\title{
«Dinge gehen vor im Mond, die das Kalb selbst nicht gewohnt»
}

Fritz Liebrich

Korrespondenz:

Dr. med. Fritz Liebrich Schaffhauserstrasse 136 CH-8302 Kloten
Im Januar 1992 konsultierte mich eine damals 42jährige Patientin wegen eines grippalen Infekts und Knieschmerzen. Zur Abklärung verordnete ich Blutuntersuchungen, und die Patientin wünschte von sich aus einen HIV-Test. Bei der nächsten Konsultation konnte ich der Patientin bekanntgeben, dass keine HIV-Antikörper nachgewiesen wurden. Sie war zufrieden, fragte mich aber nach dem HIV-Status eines meiner Patienten. Von diesem, einem Kenianer, hatte sie ein Kind, lebte im Moment der genannten Blutuntersuchung aber an einer anderen Adresse als er.

Über diesen Ex-Partner hörte ich von der Patientin nie ein freundliches Wort, es war zwischen dem Paar mehrfach zu Handgreiflichkeiten gekommen, und ich konnte mir nicht vorstellen, dass die Patientin mit diesem Mann wieder eine Beziehung aufnehmen würde. Ich orientierte die Patientin, dass ich ans Arztgeheimnis gebunden sei und sie bei Bedarf diesen Partner persönlich nach seinem HIV-Status fragen sollte. Er wusste darüber Bescheid. Er war positiv. Im Herbst 1993 spendete die Patientin Blut. Man fand bei ihr HIV-Antikörper. Eine Kollegin vom Blutspendedienst meldete mir das telefonisch, und ich drückte mein Bedauern über den Vorfall aus. Die Patientin konsultierte mich anschliessend persönlich, wobei ich das Geschehene wiederum bedauerte, allerdings nicht, ohne anzufügen, dass ich dies nicht hätte vermeiden können.

1994 kreuzte die Patientin mit einer Anwältin in meiner Praxis auf. Die Anwältin erklärte, dass ich am HIV-Infekt der Patientin wegen verweigerter Information mitschuldig sei und sie eine Schadenersatzsumme von Fr. 100 000.- einfordern werde. Ich hätte mich beim Kantonsarzt von der ärztlichen Schweigepflicht entbinden lassen müssen.

Lange hörte ich darauf zum Glück nichts mehr. Angeklagt wurde der Partner der Patientin. In einem Geschworenenprozess wurde er für schuldig befunden, eine lebensgefährliche Krankheit übertragen zu haben, und wurde deswegen mit Freiheitsentzug bestraft. Zusätzlich wurde er zu einer Genugtuung von Fr. 100 000.- verpflich- tet. Er hatte kein Geld. Für solche Fälle gäbe es eine Opferhilfe, es wurde aber von den Anwälten versäumt, sich an diese Stelle zu wenden. Im Jahr 2000, als ich den Fall beinahe vergessen hatte, bekam ich wieder eine Vorladung zu einer Einvernahme. Diesmal lautete der Vorwurf gegen mich: vorsätzliche schwere Körperverletzung. Ein Fahrlässigkeitsdelikt verjährt bereits nach 10 Jahren. Es folgten nicht enden wollende Einvernahmen von mir und von Zeugen. Wichtige Teile der Krankengeschichten der beiden Partner wurden beschlagnahmt.

Natürlich machte ich Kopien. Zusätzlich erschienen eines Tages zwei Polizeibeamte mit einem Hausdurchsuchungsbefehl in meiner Praxis. Sie forderten meine Einschreibagenda von 1992. Dies, weil die Patientin behauptet hatte, sie hätte 1992 sich und ihren Partner zu einem HIV-Test angemeldet, und der Partner hätte sich wieder abgemeldet. Um dies zu beweisen, benötigte die Polizei das Einschreibebuch. Ich gab es natürlich heraus.

Vom Gegenanwalt wurde ich folgendermassen unter Druck gesetzt:

- Erstens mit dem Strafverfahren wegen vorsätzlicher schwerer Körperverletzung. Sollte es zu einer Anklage kommen, wäre ein Geschworenenprozess die Folge gewesen. Solche Prozesse werden gegen Schwerverbrecher geführt. Mein Vorsatz bestand darin, dass ich durch die Nichtinformation der Patientin in Kauf genommen hätte, dass diese sich mit HIV infizieren würde.

- Zweitens strengte der Anwalt ein Zivilverfahren an und forderte eine Entschädigung von einer Million Franken. Diese Summe berechnete sich aus dem Einkommensverlust der Patientin. Zusätzlich veranlasste der Anwalt bei der Invalidenversicherung und der Krankenkasse der Patientin Regressforderungen. Ich war angeblich schuld an der Krankheit der Patientin und sie bezog deswegen eine IVRente. Auch Krankenkassenkosten hätte man sich logischerweise bei Nichtinfektion sparen können. Darüber hinaus wurde ich in der Klageschrift zum Zivilverfahren beschuldigt, für die Drogenkarriere der Tochter der Patien- 
tin verantwortlich zu sein. Diese Tochter sei durch die HIV-Infektion der Mutter derart belastet gewesen, dass ihr nur der Ausweg in die Drogen geblieben sei.

- Drittens setzte mich der Gegenanwalt mit der Drohung unter Druck, er bringe den Fall in die Presse und dies wäre für mich als Arzt verheerend.

- Schliesslich wurde im vergangenen Jahr ein Vergleich in Höhe von Fr. 300 000.- offeriert. Dieser wurde von der Gegenseite abgelehnt. Das Verfahren konnte Ende des letzten Jahres doch noch aussergerichtlich geregelt werden. Meine Versicherung zeigte sich sehr grosszügig.

Weniger grosszügig war die Versicherung bei der Übernahme der Anwaltskosten. Eine Haftpflichtversicherung bezahlt nichts, wenn ein Vorsatzdelikt zur Diskussion steht. Bis die Versicherungsverantwortlichen diesen Passus in ihren Bestimmungen bemerkt hatten, dauerte es recht lange. Aber eines Tages teilte man mir mit, ich hätte die $\mathrm{ab}$ einem bestimmten Datum anfallenden Anwalts- und Verfahrenskosten selbst zu tragen.

Während ich also mit meinem eigenen Ersparten weiterkämpfen musste, konnte sich die Klägerin ein langes Verfahren leisten. Sie hatte unentgeltlichen Rechtsbeistand. Ihr Anwalt wusste dies zu nutzen.

Im ganzen Verfahren hatte ich nie das Gefühl, dass in der Rechtsprechung das Prinzip gilt: im Zweifel für den Angeklagten. Für den Gegenanwalt galt die Devise: im Zweifel für den Kläger. So wurde mir vorgeworfen, ich hätte an Kran- kengeschichten und Praxisagenda manipuliert, um mich zu entlasten. Während der Einvernahmen konnte ich in Erfahrung bringen, dass die Patientin bereits zwei Monate vor dem HIVTest die Beziehung zu ihrem ehemaligen Partner wieder aufgenommen hatte und somit zum Zeitpunkt des Tests wahrscheinlich bereits angesteckt war! Zum Schluss musste mir meine Anwältin mitteilen, dass der Gegenanwalt den Vergleich davon abhängig machte, dass unsere Gemeinde auf einen Teil ihrer berechtigten Regressforderungen verzichtete. Die Patientin hat einiges an Sozialleistungen bezogen.

Natürlich fragte ich die involvierten Anwälte, was gewesen wäre, wenn ich die Patientin über den HIV-Status des Ex-Partners orientiert hätte und dieser mich wegen Verletzung des Arztgeheimnisses angeklagt hätte. Die Antwort war einhellig: «Da hätten Sie ohne weiteres einen Freispruch erhalten.» Nach allem Erfahrenen glaube ich das nicht. Schliesslich wurde im vergangenen Jahr Franco Cavalli zu einer Busse verurteilt, weil er einen Ehepartner über das Krebsleiden seiner Partnerin ohne deren Einverständnis orientiert hatte. Ein Gynäkologe wurde gebüsst, weil er eine Patientin ohne Entbindung vom Arztgeheimnis betrieben hatte. Auch heute würde ich die Patientin keinesfalls über den HIVStatus ihres angeblichen Partners orientieren. Ich wusste 1994 nicht, dass man sich in gewissen Fällen vom Kantonsarzt vom Arztgeheimnis entbinden lassen kann. Das käme für mich in diesem Fall auch heute nicht in Frage. Das Problem müssen die Partner selbst regeln. Wenn sie ihm misstraut, soll sie ihn verlassen oder sich schützen. 